\title{
Is it time to consider the sentinel lymph node mapping the new standard in endometrial cancer?
}

\author{
Andrea Papadia, Maria Luisa Gasparri, Michael D. Mueller \\ Department of Obstetrics and Gynecology, University Hospital of Bern, University of Bern, Bern, Switzerland \\ Correspondence to: Andrea Papadia, MD, PhD. Department of Obstetrics and Gynecology, University Hospital of Bern, University of Bern, \\ Effingerstrasse 102, Bern 3010, Switzerland. Email: andrea.papadia@insel.ch. \\ Comment on: Rossi EC, Kowalski LD, Scalici J, et al. A comparison of sentinel lymph node biopsy to lymphadenectomy for endometrial cancer \\ staging (FIRES trial): a multicentre, prospective, cohort study. Lancet Oncol 2017;18:384-92.
}

Submitted Apr 07, 2017. Accepted for publication Apr 17, 2017.

doi: $10.21037 /$ tcr.2017.05.10

View this article at: http://dx.doi.org/10.21037/tcr.2017.05.10

\section{Introduction}

In the march issue of Lancet Oncology, Rossi et al. published the results of a prospective multicenter validation trail on robotic indocyanine green (ICG) sentinel lymph node (SLN) mapping in endometrial cancer (EC): the FIRES trial (1). In this trial, at 18 institutions, 340 patients with EC underwent a robotic ICG SLN mapping followed by a systematic pelvic lymph node dissection in every case and by a para-aortic lymph node dissection in $58 \%$ of the cases. The median number of SLNs and of total nodes removed was 2 and 19 respectively. We believe that this is a landmark trial that may lead to changes in the management recommendations for EC. The prospective multicentre design and the strong validation data derived from its large cohort of patients and the thoroughness of the lymphadenectomy represent its major strengths.

Although multiple series have previously been published on SLN mapping in EC, this is only the second prospective validation trial on this topic. The first one is the French SENTIENDO trial in which 125 patients underwent SLN mapping with intracervical injection of blue dye and Tc99 followed by SLN biopsy and pelvic lymph node dissection; a paraaortic lymph node dissection was performed in $12 \%$ of the cases (2).

The SLN mapping in EC is a very contemporary and controversial topic which is still surrounded by skepticism and some degree of resistance in the scientific community. This is clearly documented by the heterogeneity of the recommendations on the adoption of this technique in clinical practice provided by different guidelines. According to the NCCN guidelines, SLN mapping is an acceptable procedure in selected cases since 2014, whereas according to the ESMO-ESGO-ESTRO consensus conference it should only be offered in the setting of clinical trials $(3,4)$.

\section{Surgical lymph node assessment in EC}

The pelvic and paraaortic lymphadenectomy has been a controversial topic in EC patients since it became part of the FIGO surgical staging in 1988 after the results of the seminal surgical-pathologic GOG\#33 study $(5,6)$. Ironically, 30 years later, the SLN mapping, that could represent a solomonic solution between a full lymphadenectomy and no surgical lymph nodal assessment at all, has become the new focus of the controversy. On one hand, based on the evidence derived from data of two large prospective randomized clinical trials that showed that the lymphadenectomy does not play a therapeutic role in EC, some authors argue that no form of lymph nodal resection should be performed: neither lymphadenectomy, nor SLN biopsy $(7,8)$. On the other hand, sustainers of the lymphadenectomy advocate that the validation data on this technique are few, so that its adoption in clinical practice cannot be considered safe yet.

It is impossible to reasonably talk about the surgical lymph node assessment in EC without talking about adjuvant treatment. Everybody agrees on the fact that removing grossly normal appearing lymph nodes does not have a direct therapeutic effect. However, the identification of patients with extrauterine disease is useful to select the 
patients with a poorer prognosis in order to treat them more aggressively. Similarly, EC patients who are proven to be lymph node negative may be spared an unnecessary adjuvant treatment. Although sometimes adjuvant treatment is recommended based on intrauterine risk factors, it has been proven that patients with pathologically negative lymph nodes are less frequently subjected to adjuvant therapy (mainly radiotherapy) as compared to patients in whom the lymph nodal status is unknown (9-11). For highintermediate and for high risk patients the recommendations of the ESMO-ESGO-ESTRO consensus conference differ based on the status of the lymph nodes and recommend EBRT only if it is unknown but not if the lymph nodes are negative (4). Hence, although the lymphadenectomy per se does not have a direct therapeutic effect it does have an indirect therapeutic or prognostic effect in that it helps to plan a more tailored oncologic management.

\section{False negative rate}

The single most important characteristic of the SLN mapping is the false negative rate. Only if a negative SLN can accurately predict the negativity of the non-SLNs (NSLNs) can the SLN mapping be considered a safe alternative to a systematic lymphadenectomy. Both in breast and vulvar cancer, where the SLN mapping is considered a standard treatment and has substituted a systematic lymphadenectomy, is the false negative rate approximately $3 \%(12,13)$.

\section{SLN mapping algorithm}

In 2011, the Memorial Sloan Kettering Cancer Center (MSKCC) recommended to adopt a SLN mapping algorithm whenever performing a SLN mapping in EC (14). Along with every SLN, every enlarged lymph node should be removed and, in case of unilateral SLN mapping, a side specific lymphadenectomy should be performed. Additionally, a pathological ultrastaging should be performed on the SLNs. By adopting this algorithm, Barlin et al. were able to show a significant reduction in false negative rates which dropped from $15 \%$ to $2.5 \%$ (14). In the FIRES trial, where the MSKCC SLN mapping was adopted, a false negative rate of $3 \%$ was recorded; this data is comparable to that recorded in breast and vulvar cancer $(1,12,13)$.

\section{Detection rate}

The second most important characteristic of a SLN mapping is its overall and bilateral detection rate. When applying the
MSKCC SLN mapping algorithm, high bilateral detection rates will lead to a low number of bilateral or side specific lymphadenectomies, thus optimizing the benefit in reduced morbidity offered by the SLN biopsy. One of the factors affecting the bilateral detection rate is the tracer used (15). ICG SLN mapping has been consistently shown to have higher bilateral detection rates as compared to conventional dyes after intracervical injection (16-19). In the FIRES trial, overall and bilateral detection rates were $86 \%$ and $52 \%$ respectively. This bilateral detection rate seems somewhat lower than the ones reported in other retrospective series (2024). This may be related to the fact that in the FIRES trial multiple institutions and surgeons were involved as compared to dedicated surgeons in the other series and it is plausible to hypothesize that the bilateral detection rate will increase with the experience of the surgeons. Additionally, as compared to the intracervical injection of Tc99, which is performed on the day prior to surgery without anesthesia and is followed by a lymphoscintigraphy, ICG is injected intraoperatively. This makes the procedure simpler, user friendlier and is perceived by the patients as a higher quality of care (21).

\section{Tracer injection site}

There has been some debate on the most appropriate injection site when performing a SLN mapping in EC. The uterus is drained through four lymphatic draining systems which include a higher paracervical pathway that runs along the uterine vessels to the obturator, external iliac and common iliac lymph nodes; a lower paracervical pathway that runs along the ureter to the internal iliac and presacral lymph nodes, a lymphatic drainage that runs along the round ligament to the distalmost iliac and to the inguinofemoral lymph nodes and a lymphatic pathway that follows the infundibulo pelvic ligament and drains to the high paraaortic lymph nodes (25). Some authors recommend a hysteroscopic peritumoral injection of the tracer, since this will lead to a different anatomic distribution of the SLNs (26,27). In a small prospective pilot study, Rossi et al. compared the intracervical and peritumoral ICG injection in EC patients undergoing robotic SLN mapping showing that the intracervical injection was superior achieving a higher SLN detection rate and a similar anatomic nodal distribution as hysteroscopic endometrial injection (28). Theoretically a SLN from each draining lymphatic way should be removed. However, in the majority of the patients a SLN will not be identified on each lymphatic pathway and it has been suggested that a higher number of SLNs may 
not have a significant impact on the false negative rate (29).

\section{Low risk EC}

With regards to the indication to the SLN mapping, this has been adopted in various settings although its application in high risk patients has been seen as controversial. In patients with a preoperative diagnosis of complex atypical hyperplasia $(\mathrm{CAH})$ or grade 1 or 2 endometrioid $\mathrm{EC}$ it has been shown to be more accurate in detecting stage IIIC cancer patients as compared to a strategy in which a systematic lymphadenectomy is performed based on intrauterine risk factors identified at frozen section (30,31). Patients with $\mathrm{CAH}$ have also been subjected to SLN mapping given their elevated risk of being diagnosed with an invasive EC at final pathological analysis $(32,33)$. Sinno et al. have proposed a surgical treatment algorithm in which every patient undergoes a SLN mapping, whereas the frozen section evaluation of the uterus is reserved to those patients who fail to map or who map only unilaterally; in these patients a lymphadenectomy or selective lymphadenectomy is performed based on the results of the frozen section (30). This strategy optimizes the yield of metastatic lymph nodes and reduces the number of systematic lymphadenectomies in node negative patients. Based on frozen section evaluation of the uterus more than $30 \%$ of the patients with these preoperative characteristics will undergo a lymphadenectomy but only $10 \%$ will have lymph node metastases $(30,31)$. Furthermore, patients with apparently low risk EC may end up having a high intermediate or high risk EC at final histological analysis with a risk of lymph node metastases that is as high as $40 \%$ (34). In these cases, the lack of lymph nodal information indicates to an adjuvant EBRT which would have otherwise not been given had the lymph nodal status been known to be negative (4). In these patients the knowledge of the pathological status of the lymph nodes, whether SLNs or not, becomes crucial to plan an appropriate management. Furthermore, the knowledge of the anatomic distribution of the lymph nodal metastases helps to determine the radiation fields when an EBRT is indicated (pelvic EBRT or with extended fields of irradiation to the paraaortic area).

So far, the most widespread method to triage patients to surgical staging has been via intraoperative identification of pathologic risk factors at frozen section (35-38). However, this strategy is suboptimal since it only provides the risk of lymph nodal metastases. As a consequence, no information whatsoever on lymph nodal status will be gathered in patients intraoperatively identified as low risk, whereas a significant number of systematic lymphadenectomy will still be performed in lymph node negative patients.

\section{High grade EC}

Based on the results of the SENTIENDO trial, in which all the false negative cases occurred in patients with high risk EC, the adoption of the SLN mapping in this setting has occurred with more caution (2). On the contrary, in the FIRES trial, circa 100 patients with high risk EC, defined as grade 3 or with high risk histology, were enrolled and this did not impact negatively on the false negative rate of the SLN mapping (1). Data from this large comprehensively staged cohort of high risk patients support the adoption of the SLN biopsy in high risk EC as well. Recently, in a small series published by the Duke University, robotic ICG SLN mapping performed well in patients with high risk histology (39). They reported a false negative rate that dropped from $15 \%$ to $2 \%$ after the application of the SLN mapping algorithm proposed by MSKCC. These data compare well with those reported in the FIRES trial and in other series and suggest that the SLN mapping might be safely applied in this subgroup of patients as well. Furthermore, these data support the strict adherence to the SLN algorithm to keep the false negative rate reasonably low. Alternatively, the preoperative evaluation with a PET/CT has been proposed as a triage tool in patients with high grade EC $(40,41)$. According to Signorelli et al., a SLN biopsy should be reserved to the patients with a negative PET/CT, whereas a full lymphadenectomy should be offered to patients with a PET/CT suggestive for nodal metastases (40).

\section{Pathological ultrastaging}

The pathological ultrastaging of the SLNs is part of the SLN mapping algorithm and should be offered to all the patients undergoing SLN mapping (14). It consists in a more thorough pathologic evaluation of the SLNs. Briefly, SLNs are initially examined by routine hematoxylin \& eosin ( $\mathrm{H} \& \mathrm{E})$ staining, and subsequent ultrastaging is performed if the initial $\mathrm{H} \& \mathrm{E}$ assessment is negative. This is performed by cutting 2 adjacent 5 - $\mu \mathrm{m}$ sections at each of 2 levels, $50-\mu \mathrm{m}$ apart from each paraffin block lacking metastatic carcinoma. At each level, one slide is stained with $\mathrm{H} \& \mathrm{E}$ and with immunohistochemistry (IHC) using the anti-cytokeratin AE1:AE3 (Ventana Medical Systems, Inc, Tucson, AZ, USA) for a total of 5 slides per block. According to the definition of the American Joint 
Committee on Cancer, metastatic disease to the SLNs is then classified as follows (42):

(I) Macrometastases when tumor deposits larger than $2.0 \mathrm{~mm}$ are identified;

(II) Micrometastases when tumor deposits measuring more than $0.2 \mathrm{~mm}$ and up to $2 \mathrm{~mm}$ were detected via $\mathrm{H} \& \mathrm{E}$ staining and/or and cytokeratin immunohistochemical stains (AE1:AE3);

(III) Isolated tumor cells when tumor deposits measuring up to $2 \mathrm{~mm}$ were detected via H\&E staining and/or and cytokeratin immunohistochemical stains (AE1:AE3).

The pathological ultrastaging has led to an increase in identification of small volume lymph node metastases that would otherwise go undetected (43). In the reported series, micrometastases and ITCs consistently represent circa $40-50 \%$ of the lymph node metastases (44). In the FIRES trial, micrometastases and ITC accounted for 54\% of the metastatic lymph nodes (1).

A few questions on the significance and the clinical management of these metastases remain unanswered. Firstly, it is still unclear whether micrometastases and ITCs carry the same prognostic significance as macrometastases. Secondly, it is unclear what to do if a SLN is found to be affected by disease: should a systematic lymphadenectomy be performed or not? Touhami et al. have shown that the risk of NSLNs to be involved by metastatic disease is $5 \%$ in case of micrometastases and ITC to the SLNs and raises to over $60 \%$ in case of macrometastases to the SLNs (44). In the FIRES trial, the SLN represented the most distal level (pelvic vs. para-aortic) of metastatic spread in $80 \%$ of the cases (1). Based on these results it is argued that a systematic lymphadenectomy should be considered only in patients that harbor macrometastastatic disease to the SLNs.

\section{Oncological outcome}

A few retrospective series have tried to analyze the oncologic outcome of the SLN mapping in EC patients. How et al. were able to demonstrate a reduction in pelvic lymph node recurrences in a cohort of $\mathrm{EC}$ patients undergoing SLN biopsy followed by systematic lymphadenectomy when compared to an historic cohort of patients undergoing a systematic lymphadenectomy without SLN biopsy (45). Interestingly, the group of patients experiencing less recurrence presented more often with deep myometrial invasion and LVSI. The author suggest that this result has to be attributed to the adoption of the SLN biopsy, that allows for identification of lymph nodes in areas that are not usually included in a pelvic lymphadenectomy such as the presacral and hypogastric region in $14 \%$ of the cases. Finally, in a large retrospective study from the Mayo Clinic and MSKCC involving over 1,000 patients with EC undergoing a systematic pelvic and paraaortic lymphadenectomy or a SLN mapping algorithm, excellent and comparable 3-year disease free survival rates were recorded for both groups (46).

\section{Conclusions}

The recently published FIRES trial provides solid validation data of this technique given the large number of patients enrolled and the thoroughness of the lymphadenectomy performed and we hope that this will provide a positive drive in the incorporation of the minimally invasive ICG SLN mapping as a recommended procedure in $\mathrm{EC}$ patients in the European guidelines (1). Minimally invasive ICG SLN mapping in EC with an intracervical tracer injection provides good results with a low false negative rate and high detection rates. It should be reserved to patients without apparent extrauterine metastases and a strict adherence to the MSKCC algorithm is essential. Current data to support the accuracy of the SLN mapping in detecting nodal disease in high grade $\mathrm{EC}$ are strong but still inconclusive. The clinical significance of metastatic disease detected by ultrastaging is still unclear. Similarly, the role of a completion lymphadenectomy in SLN positive patients has to be determined.

\section{Acknowledgments}

Funding: None.

\section{Footnote}

Provenance and Peer Review: This article was commissioned and reviewed by the Section Editor Zheng Li (Department of Gynecologic Oncology, The Third Affiliated Hospital of Kunming Medical University (Yunnan Tumor Hospital), Kunming, China).

Conflicts of Interest: All authors have completed the ICMJE uniform disclosure form (available at http://dx.doi. org/10.21037/tcr.2017.05.10). The authors have no conflicts of interest to declare.

Ethical Statement: The authors are accountable for all aspects of the work in ensuring that questions related 
to the accuracy or integrity of any part of the work are appropriately investigated and resolved.

Open Access Statement: This is an Open Access article distributed in accordance with the Creative Commons Attribution-NonCommercial-NoDerivs 4.0 International License (CC BY-NC-ND 4.0), which permits the noncommercial replication and distribution of the article with the strict proviso that no changes or edits are made and the original work is properly cited (including links to both the formal publication through the relevant DOI and the license). See: https://creativecommons.org/licenses/by-nc-nd/4.0/.

\section{References}

1. Rossi EC, Kowalski LD, Scalici J, et al. A comparison of sentinel lymph node biopsy to lymphadenectomy for endometrial cancer staging (FIRES trial): a multicentre, prospective, cohort study. Lancet Oncol 2017;18:384-92.

2. Ballester M, Dubernard G, Lécuru F, et al. Detection rate and diagnostic accuracy of sentinel-node biopsy in early stage endometrial cancer: a prospective multicentre study (SENTI-ENDO). Lancet Oncol 2011;12:469-76.

3. NCCN Clinical Practice Guidelines in Oncology. Uterine Neoplasms. Version 2.2014. Available online: http://www. nccn.org/professionals/physician_gls/pdf/uterine.pdf

4. Colombo N, Creutzberg C, Amant F, et al. ESMOESGO-ESTRO Consensus Conference on Endometrial Cancer: diagnosis, treatment and follow-up. Ann Oncol 2016;27:16-41.

5. FIGO stages: 1988 revision. Gynecol Oncol 1989;35:125-7.

6. Creasman WT, Morrow CP, Bundy BN, et al. Surgical pathologic spread patterns of endometrial cancer. A Gynecologic Oncology Group Study. Cancer 1987;60:2035-41.

7. Benedetti Panici P, Basile S, Maneschi F, et al. Systematic pelvic lymphadenectomy vs. no lymphadenectomy in early-stage endometrial carcinoma: randomized clinical trial. J Natl Cancer Inst 2008;100:1707-16.

8. ASTEC study group, Kitchener H, Swart AM, et al. Efficacy of systematic pelvic lymphadenectomy in endometrial cancer (MRC ASTEC trial): a randomised study. Lancet 2009;373:125-36.

9. Simpkins F, Papadia A, Kunos C, et al. Patterns of recurrence in stage I endometrioid endometrial adenocarcinoma with lymphovascular space invasion. Int J Gynecol Cancer 2013;23:98-104.

10. Bogani G, Dowdy SC, Cliby WA, et al. Role of pelvic and para-aortic lymphadenectomy in endometrial cancer: current evidence. J Obstet Gynaecol Res 2014;40:301-11.

11. Sharma C, Deutsch I, Lewin SN, et al.
Lymphadenectomy influences the utilization of adjuvant radiation treatment for endometrial cancer. Am J Obstet Gynecol 2011;205:e1-9.

12. Veronesi U, Paganelli G, Viale G, et al. A randomized comparison of sentinel-node biopsy with routine axillary dissection in breast cancer. $\mathrm{N}$ Engl $\mathrm{J}$ Med 2003;349:546-53.

13. Van der Zee AG, Oonk MH, De Hullu JA, et al. Sentinel node dissection is safe in the treatment of early-stage vulvar cancer. J Clin Oncol 2008;26:884-9.

14. Barlin JN, Khoury-Collado F, Kim CH, et al. The importance of applying a sentinel lymph node mapping algorithm in endometrial cancer staging: beyond removal of blue nodes. Gynecol Oncol 2012;125:531-5.

15. Ruscito I, Gasparri ML, Braicu EI, et al. Sentinel node mapping in cervical and endometrial cancer: indocyanine green versus other conventional Dyes-A Meta-Analysis. Ann Surg Oncol 2016;23:3749-56.

16. Imboden S, Papadia A, Nauwerk M, et al. A comparison of radiocolloid and indocyanine green fluorescence imaging, sentinel lymph node mapping in patients with cervical cancer undergoing laparoscopic surgery. Ann Surg Oncol 2015;22:4198-203.

17. Tanner EJ, Sinno AK, Stone RL, et al. Factors associated with successful bilateral sentinel lymph node mapping in endometrial cancer. Gynecol Oncol 2015;138:542-7.

18. Buda A, Papadia A, Zapardiel I, et al. From conventional radiotracer Tc-99(m) with blue dye to indocyanine green fluorescence: a comparison of methods towards optimization of sentinel lymph node mapping in early stage cervical cancer for a laparoscopic approach. Ann Surg Oncol 2016;23:2959-65.

19. Papadia A, Zapardiel I, Bussi B, et al. Sentinel lymph node mapping in patients with stage I endometrial carcinoma: a focus on bilateral mapping identification by comparing radiotracer $\mathrm{Tc} 99(\mathrm{~m})$ with blue dye versus indocyanine green fluorescent dye. J Cancer Res Clin Oncol 2017;143:475-80.

20. Papadia A, Imboden S, Siegenthaler FA, et al. Laparoscopic indocyanine green sentinel lymph node mapping in endometrial cancer. Ann Surg Oncol 2016;23:2206-11.

21. Buda A, Elisei F, Palazzi S, et al. Quality of Care for Cervical and Endometrial Cancer Patients: The Impact of Different Techniques of Sentinel Lymph Node Mapping on Patient Satisfaction. Ann Surg Oncol 2016;23:2975-81.

22. Holloway RW, Bravo RA, Rakowski JA, et al. Detection of sentinel lymph nodes in patients with endometrial cancer undergoing robotic-assisted staging: a comparison of colorimetric and fluorescence imaging. Gynecol Oncol 2012;126:25-9.

23. Plante $M$, Touhami $O$, Trinh XB, et al. Sentinel node mapping with indocyanine green and endoscopic near-infrared fluorescence imaging in endometrial cancer. A pilot study and 
review of the literature. Gynecol Oncol 2015;137:443-7.

24. Jewell EL, Huang JJ, Abu-Rustum NR, et al. Detection of sentinel lymph nodes in minimally invasive surgery using indocyanine green and near-infrared fluorescence imaging for uterine and cervical malignancies. Gynecol Oncol 2014;133:274-7.

25. Geppert B, Lönnerfors C, Bollino M, et al. A study on uterine lymphatic anatomy for standardization of pelvic sentinel lymph node detection in endometrial cancer. Gynecol Oncol 2017;145:256-61.

26. Ditto A, Martinelli F, Bogani G, et al. Sentinel node mapping using hysteroscopic injection of indocyanine green and laparoscopic Near-Infrared fluorescence imaging in endometrial cancer staging. J Minim Invasive Gynecol 2015;22:132-3.

27. Martinelli F, Ditto A, Bogani GA, et al. Laparoscopic sentinel node mapping in endometrial cancer after hysteroscopic injection of indocyanine green. J Minim Invasive Gynecol 2017;24:89-93.

28. Rossi EC, Jackson A, Ivanova A, et al. Detection of sentinel nodes for endometrial cancer with robotic assisted fluorescence imaging: cervical versus hysteroscopic injection. Int J Gynecol Cancer 2013;23:1704-11.

29. Papadia A, Imboden S, Gasparri ML, et al. Endometrial and cervical cancer patients with multiple sentinel lymph nodes at laparoscopic ICG mapping: How many are enough? J Cancer Res Clin Oncol 2016;142:1831-6.

30. Sinno AK, Peijnenburg E, Fader AN, et al. Reducing overtreatment: A comparison of lymph node assessment strategies for endometrial cancer. Gynecol Oncol 2016;143:281-6.

31. Papadia A, Gasparri ML, Siegenthaler F, et al. FIGO stage IIIC endometrial cancer identification among patients with complex atypical hyperplasia, grade 1 and 2 endometrioid endometrial cancer: laparoscopic indocyanine green sentinel lymph node mapping versus frozen section of the uterus, why get around the problem? J Cancer Res Clin Oncol 2017;143:491-7.

32. Morotti M, Menada MV, Moioli M, et al. Frozen section pathology at time of hysterectomy accurately predicts endometrial cancer in patients with preoperative diagnosis of atypical endometrial hyperplasia. Gynecol Oncol 2012;125:536-40.

33. Trimble CL, Kauderer J, Zaino R, et al. Concurrent endometrial carcinoma in women with a biopsy diagnosis of atypical endometrial hyperplasia - A Gynecologic Oncology Group Study. Cancer 2006;106:812-9.

34. Ouldamer L, Bendifallah S, Body G, et al. Call for Surgical Nodal Staging in Women with ESMO/ESGO/ ESTRO High-Intermediate Risk Endometrial Cancer: A Multicentre Cohort Analysis from the FRANCOGYN Study Group. Ann Surg Oncol 2017;24:1660-6.
35. Papadia A, Azioni G, Brusacà B, et al. Frozen section underestimates the need for surgical staging in endometrial cancer patients. Int J Gynecol Cancer 2009;19:1570-3.

36. Kumar S, Medeiros F, Dowdy SC, et al. A prospective assessment of the reliability of frozen section to direct intraoperative decision making in endometrial cancer. Gynecol Oncol 2012;127:525-31.

37. Laufer J, Scasso S, Papadia A, et al. Association between tumor diameter and lymphovascular space invasion among women with early-stage endometrial cancer. Int J Gynaecol Obstet 2013;123:142-5.

38. Sala P, Morotti M, Menada MV, et al. Intraoperative frozen section risk assessment accurately tailors the surgical staging in patients affected by early-stage endometrial cancer: the application of 2 different risk algorithms. Int J Gynecol Cancer 2014;24:1021-6.

39. Ehrisman J, Secord AA, Berchuck A, et al. Performance of sentinel lymph node biopsy in high-risk endometrial cancer. Gynecol Oncol Rep 2016;17:69-71.

40. Signorelli M, Crivellaro C, Buda AA, et al. Staging of High-Risk endometrial cancer with PET/CT and sentinel lymph node mapping. Clin Nucl Med 2015;40:780-5.

41. Bese T, Sal V, Demirkiran F, et al. The Combination of Preoperative Fluorodeoxyglucose Positron Emission Tomography/Computed Tomography and Sentinel Lymph Node Mapping in the Surgical Management of Endometrioid Endometrial Cancer. Int J Gynecol Cancer 2016;26:1228-38.

42. Greene FL, Page DL, Irvin D, et al. editors. AJCC Cancer Staging Manual. 6th ed. New York: Springer-Verlag, 2003:223-40.

43. Kim CH, Soslow RA, Park KJ, et al. Pathologic ultrastaging improves micrometastasis detection in sentinel lymph nodes during endometrial cancer staging. Int J Gynecol Cancer 2013;23:964-70.

44. Touhami O, Trinh XB, Gregoire J, et al. Predictors of non-sentinel lymph node (non-SLN) metastasis in patients with sentinel lymph node (SLN) metastasis in endometrial cancer. Gynecol Oncol 2015;138:41-5.

45. How J, Gauthier C, Abitbol J, et al. Impact of sentinel lymph node mapping on recurrence patterns in endometrial cancer. Gynecol Oncol 2017;144:503-9.

46. Eriksson AG, Ducie J, Ali N, et al. Comparison of a sentinel lymph node and a selective lymphadenectomy algorithm in patients with endometrioid endometrial carcinoma and limited myometrial invasion. Gynecol Oncol 2016;140:394-9.

Cite this article as: Papadia A, Gasparri ML, Mueller MD. Is it time to consider the sentinel lymph node mapping the new standard in endometrial cancer? Transl Cancer Res 2017;6(Suppl 3):S547-S552. doi: 10.21037/tcr.2017.05.10 